Research Article

\title{
Impulse Propagation in Compositions and Words
}

\author{
Margaret Archibald $\mathbb{D},{ }^{1}$ Aubrey Blecher $\mathbb{D}^{1},{ }^{1}$ Charlotte Brennan $\mathbb{D}^{1}{ }^{1}$ \\ Arnold Knopfmacher $\mathbb{D}^{1},{ }^{1}$ and Toufik Mansour ${ }^{2}{ }^{2}$ \\ ${ }^{1}$ The John Knopfmacher Centre for Applicable Analysis and Number Theory, University of the Witwatersrand, P. O. Wits, 2050, \\ Johannesburg, South Africa \\ ${ }^{2}$ Department of Mathematics, University of Haifa, 199 Abba Khoushy Ave 3498838, Haifa, Israel
}

Correspondence should be addressed to Charlotte Brennan; charlotte.brennan@wits.ac.za

Received 26 August 2020; Revised 10 December 2020; Accepted 18 December 2020; Published 21 January 2021

Academic Editor: Sergejs Solovjovs

Copyright (c) 2021 Margaret Archibald et al. This is an open access article distributed under the Creative Commons Attribution License, which permits unrestricted use, distribution, and reproduction in any medium, provided the original work is properly cited.

\begin{abstract}
We consider compositions of $n$ represented as bargraphs and subject these to repeated impulses which start from the left at the top level and destroy horizontally connected parts. This is repeated while moving to the right first and then downwards to the next row and the statistic of interest is the number of impulses needed to annihilate the whole composition. We achieve this by conceptualizing a generating function that tracks compositions as well as the number of impulses used. This conceptualization is repeated for words (over a finite alphabet) represented by bargraphs.
\end{abstract}

\section{Introduction}

In several recent papers modelling physical or chemical situations, molecules and other structures are represented by combinatorial objects (e.g., compositions) and various physical entities (such as light or force) acting on the objects are modelled in the mathematical context of these combinatorial objects (see [1-4]). In this paper, we continue this project using bargraphs (see [5]) to model the physical objects subject to impulses which destroy them. An impulse starts from the left at the top level and destroys the blocks formed by the horizontally connected cells. The number of such impulses is the focus of attention. Each block on a given level requires a separate impulse to destroy it. Bargraphs are nonintersecting lattice paths in $\mathbb{N}_{0}^{2}$ with three allowed types of steps; up $(0,1)$, down $(0,-1)$, and horizontal $(1,0)$. They start at the origin with an up step and terminate immediately upon return to the $x$-axis. Bargraphs can represent compositions and words where the respective height of each horizontal step corresponds to the respective size of each part of the three structures. A composition is a bargraph of area $n$. It is well known that there are precisely $2^{n-1}$ compositions of size (or area) $n$ see Figure 1 .
The impulses on the same horizontal level have the same color. There are two impulses on level 2.

In what follows, we devote one section to each of the combinatorial structures (compositions and words). Each section develops the generating function for the number of impulses required to annihilate the respective structures, and we obtain the average number of such impulses required for each of the structures in relation to its defining parameter (size of the composition and length of words). In Section 4, we also study the number of impulses at level $j$ in these bargraphs.

\section{Compositions}

Compositions of $n$ (see [6]) are finite sequences of positive integers $\left(\sigma_{i}\right)_{i=1}^{k}$ such that

$$
\sigma_{1}+\sigma_{2}+\cdots+\sigma_{k}=n
$$

where the $\sigma_{i}$ are called parts and denote the height of the columns in the associated bargraph.

The generating function $P(x, y, u)$ (see [7]) will count compositions where $x$ marks the size of the composition $n, y$ is the number of parts, and $u$ is the number of impulses. 
We shall consider $P(x, y, u \mid a)$, the generating function for compositions where the first part is of size $a$. We decompose this situation into three cases: namely, where the composition has only one part (of size $a$ ), or for more than one part, we take compositions starting with a part of size $b$ and prepend a part of size $a$ to the left with $a<b$ or else we prepend a part $a \geq b$. We sum the three respective generating functions:

$$
\begin{aligned}
P(x, y, u \mid a)= & x^{a} y u^{a}+\sum_{a \leq b} x^{a} y P(x, y, u \mid b) \\
& +\sum_{a>b} x^{a} y u^{a-b} P(x, y, u \mid b) .
\end{aligned}
$$

For convenience, suppress the other variables and introduce variable $v$ that tracks the size of the first letter. So, we define

$$
P(v):=P(x, y, u, v)=1+\sum_{a \geq 1} P(x, y, u \mid a) v^{a}
$$

Thus,

$$
\begin{aligned}
P(v) & =1+\sum_{a \geq 1} x^{a} y u^{a} v^{a}+\sum_{a \geq 1} \sum_{a \leq b} x^{a} y P(x, y, u \mid b) v^{a}+\sum_{a \geq 1} \sum_{a>b} x^{a} y u^{a-b} P(x, y, u \mid b) v^{a} \\
& =1+\frac{x y u v}{1-x u v}+y \sum_{b \geq 1} \sum_{a=1}^{b} x^{a} v^{a} P(x, y, u \mid b)+y \sum_{b \geq 1} \sum_{a=b+1}^{\infty} x^{a} u^{a-b} v^{a} P(x, y, u \mid b) \\
& =1+\frac{x y u v}{1-x u v}+y \sum_{b \geq 1} x v \frac{1-(x v)^{b}}{1-x v}+y \sum_{b \geq 1} u^{-b} P(x, y, u \mid b) \frac{(x u v)^{b+1}}{1-x u v} \\
& =1+\frac{x y u v}{1-x u v}+y \sum_{b \geq 1} \frac{x v-(x v)^{b+1}}{1-x v}+y \sum_{b \geq 1} u P(x, y, u \mid b) \frac{(x v)^{b+1}}{1-x u v} \\
& =1+\frac{x y u v}{1-x u v}+\frac{x y v}{1-x v}[(P(1)-1)-(P(x v)-1)]+\frac{x y u v}{1-x u v}[P(x v)-1] \\
& =1+\frac{x y u v}{1-x u v}+\frac{x y v}{1-x v} P(1)-\frac{x y u v}{1-x u v}+P(x v)\left[\frac{x y u v}{1-x u v}-\frac{x y v}{1-x v}\right] \\
& =1+\frac{x y v}{1-x v} P(1)+P(x v) \frac{x y v(u-1)}{(1-x u v)(1-x v)}
\end{aligned}
$$

This is an expression that can be iterated. For simplicity, we define $A(v):=(x y v(u-1) /((1-x u v)(1-x v)))$. Thus,

$$
\begin{aligned}
P(v)= & 1+\frac{x y v}{1-x v} P(1)+A(v)\left[1+\frac{x^{2} y v}{1-x^{2} v} P(1)+P\left(x^{2} v\right) A(x v)\right] \\
= & 1+A(v)+\left[\frac{x y v}{1-x v}+A(v) \frac{x^{2} y v}{1-x^{2} v}\right] P(1) \\
& +A(v) A(x v)\left[1+\frac{x^{3} y v}{1-x^{3} v} P(1)+P\left(x^{3} v\right) \frac{x^{3} y v(u-1)}{\left(1-x^{3} u v\right)\left(1-x^{3} v\right)}\right] \\
= & 1+A(v)+A(v) A(x v)+\left[\frac{x y v}{1-x v}+A(v) \frac{x^{2} y v}{1-x^{2} v}+A(v) A(x v) \frac{x^{3} y v}{1-x^{3} v}\right] P(1) \\
& +A(v) A(x v) A\left(x^{2} v\right) P\left(x^{3} v\right) .
\end{aligned}
$$


Carrying on this process ad infinitum, we obtain

$$
\begin{aligned}
P(v)= & +\sum_{j \geq 0} \prod_{i=0}^{j} A\left(x^{i} v\right)+P(1) \sum_{j \geq 0} \frac{y x^{j+1} v}{1-x^{j+1} v} \prod_{i=0}^{j-1} A\left(x^{i} v\right) \\
= & 1+\sum_{j \geq 0} \prod_{i=0}^{j} \frac{x^{i+1} y v(u-1)}{\left(1-x^{i+1} u v\right)\left(1-x^{i+1} v\right)}+P(1) \sum_{j \geq 0} \frac{y x^{j+1} v}{1-x^{j+1} v} \prod_{i=0}^{j-1} \frac{x^{i+1} y v(u-1)}{\left(1-x^{i+1} u v\right)\left(1-x^{i+1} v\right)} \\
= & 1+\sum_{j \geq 0}(y v(u-1))^{j+1} \prod_{i=0}^{j} \frac{x^{i+1}}{\left(1-x^{i+1} u v\right)\left(1-x^{i+1} v\right)} \\
& +P(1) \sum_{j \geq 0} \frac{y x^{j+1} v}{1-x^{j+1} v}(y v(u-1))^{j} \prod_{i=0}^{j-1} \frac{x^{i+1}}{\left(1-x^{i+1} u v\right)\left(1-x^{i+1} v\right)} .
\end{aligned}
$$

Now, substituting $v=1$ yields

$$
P(1)=1+\sum_{j \geq 0}(y(u-1))^{j+1} \prod_{i=0}^{j} \frac{x^{i+1}}{\left(1-x^{i+1} u\right)\left(1-x^{i+1}\right)}+P(1) \sum_{j \geq 0} \frac{y x^{j+1}}{1-x^{j+1}}(y(u-1))^{j} \prod_{i=0}^{j-1} \frac{x^{i+1}}{\left(1-x^{i+1} u\right)\left(1-x^{i+1}\right)}
$$

Thus, we can state the following result.

Theorem 1. We have

$$
P(x, y, u)=1+\sum_{j \geq 0}(y(u-1))^{j+1} \frac{\prod_{i=0}^{j}\left(x^{i+1} /\left(\left(1-x^{i+1} u\right)\left(1-x^{i+1}\right)\right)\right)}{1-\sum_{j \geq 0}\left(\left(y x^{j+1}\right) /\left(1-x^{j+1}\right)\right)(y(u-1))^{j} \prod_{i=0}^{j-1}\left(x^{i+1} /\left(\left(1-x^{i+1} u\right)\left(1-x^{i+1}\right)\right)\right) .}
$$

For example, in Theorem 1 with $u=1$, we get back the well-known generating function for all compositions:

$$
P(x, y, 1,1)=\frac{1}{1-(y x /(1-x))}=\frac{1-x}{1-x-x y},
$$

where the size of the composition is counted by $x$ and the number of parts is counted by $y$.

Theorem 1 shows that

$$
\begin{aligned}
\left.\frac{\mathrm{d}}{\mathrm{d} u} P(x, y, u)\right|_{u=1} & =\frac{\left(x y(1-x)^{2}\right)\left(1-\left(y x /\left(1-x^{2}\right)\right)\right)}{(1-(y x /(1-x)))^{2}} \\
& =\frac{x y\left(1-x^{2}-y x\right)}{\left(1-x^{2}\right)(1-x-y x)^{2}},
\end{aligned}
$$

which leads to

$$
\begin{aligned}
\left.\frac{\mathrm{d}}{\mathrm{d} u} P(x, 1, u)\right|_{u=1} & =\frac{x\left(1-x-x^{2}\right)}{\left(1-x^{2}\right)(1-2 x)^{2}} \\
& =\sum_{n \geq 0} \frac{(3 n+10) 2^{n}-9-(-1)^{n}}{18} x^{n}
\end{aligned}
$$

Thus, we can state the following result.

Corollary 1. The total number of impulses over all compositions of $n$ is given by

$$
\frac{(3 n+10) 2^{n}-9-(-1)^{n}}{18} .
$$

Dividing by $2^{n-1}$, we find that the average number of impulses to destroy a composition of size $n$ is

$$
\frac{n}{3}+\frac{10}{9}+O\left(2^{-n}\right) \text { as } n \longrightarrow \infty \text {. }
$$




\section{Words}

Words over the alphabet $[k]$ are finite sequences $a_{1} a_{2} \ldots a_{n}$, where each $a_{i} \in[k]$ (see [6]). For the purposes of this paper, we represent each such word as a bargraph (see the previous section) with maximum column height $k$.

The generating function $W_{k}(x, u)$ will count words over the alphabet $[k]$ of length $n$ (bargraphs with $n$ columns and the height of each column is at most $k$ ), where $x$ marks the size of the words $n$ and $u$ is the number of impulses. Let $W_{k}(x, u \mid a)$ be the generating function for such words where the first part is of size $a$. By (2) and the fact that $W_{k}(x, u)=1+\sum_{a=1}^{k} W_{k}(x, u \mid a)$, we have

$$
W_{k}(x, u \mid a)=x u^{a}+x \sum_{b=a}^{k} W_{k}(x, u \mid b)+x \sum_{b=1}^{a-1} u^{a-b} W_{k}(x, u \mid b)=x\left(u^{a}-1\right)+x W_{k}(x, u)+x \sum_{b=1}^{a-1}\left(u^{a-b}-1\right) W_{k}(x, u \mid b) .
$$

By induction on $a$, we obtain

$$
W_{k}(x, u \mid a)=\sum_{\pi_{1} \pi_{2} \cdots \pi_{m} \in C_{a}} x^{m}\left(u^{\pi_{1}}-1+W_{k}(x, u)\right) \prod_{j=2}^{m}\left(u^{\pi_{j}}-1\right)
$$

where $C_{a}$ is the set of compositions of $a$. Actually, $\sum_{\pi_{1} \ldots \pi_{m} \in C_{a}}$ means $\sum_{m} \sum_{\pi_{1} \ldots \pi_{m} \in C_{a, m}}$. Thus,

$$
W_{k}(x, u)-1=\sum_{a=1}^{k} W_{k}(x, u \mid a)=\sum_{a=1}^{k} \sum_{\pi_{1} \pi_{2} \cdots \pi_{m} \in C_{a}} x^{m}\left(u^{\pi_{1}}-1+W_{k}(x, u)\right) \prod_{j=2}^{m}\left(u^{\pi_{j}}-1\right)
$$

which implies the following theorem.

Theorem 2. We have

$$
W_{k}(x, u)=1+\sum_{a=1}^{k} \sum_{\pi_{1} \pi_{2} \cdots \pi_{m} \in C_{a}} x^{m} \frac{\prod_{j=1}^{m}\left(u^{\pi_{j}}-1\right)}{1-\sum_{a=1}^{k} \sum_{\pi_{1} \pi_{2} \cdots \pi_{m} \in C_{a}} x^{m} \prod_{j=2}^{m}\left(u^{\pi_{j}}-1\right) .}
$$

In particular, $W_{k}(x, 1)=1 /\left(1-\sum_{a=1}^{k} x\right)=1 /(1-k x)$, which is the well-known generating function for the number of words of length $n$ over the alphabet $[k]$.

Differentiating the equation in Theorem 2 yields

$$
\left.\frac{\mathrm{d}}{\mathrm{d} u} W_{k}(x, u)\right|_{u=1}=\frac{x}{1-k x}\left(\begin{array}{c}
k+1 \\
2
\end{array}\right)+\frac{x}{(1-k x)^{2}}\left(\begin{array}{c}
k+1 \\
3
\end{array}\right) \text {, }
$$

which leads to the following corollary.

Corollary 2. The total number of impulses for all words of length $n$ over alphabet $[k]$ is given by

$$
\left(\left(\begin{array}{c}
k+1 \\
2
\end{array}\right)+n\left(\begin{array}{c}
k+1 \\
3
\end{array}\right)\right) k^{n-1}
$$

Hence, the average number of impulses for a word of length $n$ is $\left(\left(k^{2}-1\right) / 6\right) n+((k+1) / 2)$.

\section{Impulses at Level $j$}

Define $I_{j}(\pi)$ to be the number of impulses at level $j$ in the bargraph $\pi$. For instance, $I_{1}(\pi)=1, I_{2}(\pi)=2, I_{3}(\pi)=1$, $I_{4}(\pi)=1$, and $I_{j}(\pi)=0$ for all $j \geq 5$, where $\pi$ is given in Figure 1. Define $H\left(x, y ; q_{1}, q_{2}, \ldots\right)$ to be the generating function for the number of compositions of $n$ with exactly $m$ parts and number of impulses at level $j$, that is,

$$
H\left(x, y ; q_{1}, q_{2}, \ldots\right)=\sum_{n \geq 0} \sum_{m=0}^{n} x^{n} y^{m} \sum_{\pi} \prod_{j \geq 0} q_{j}^{I_{j}(\pi)},
$$

where $\sum_{\pi}$ is sum over all compositions of $n$ with exactly $m$ parts.

Let us write an equation for $H\left(x, y ; q_{1}, q_{2}, \ldots\right)$. Note that each composition $\pi=\pi_{1} \pi_{2} \cdots \pi_{m}$ can be decomposed as follows: it is either empty; or $\pi=1$; or $\pi_{1}=1$ and $m \geq 2$; or $\pi_{1}, \ldots, \pi_{m} \geq 2$ and $m \geq 1$; or $\pi_{1}, \ldots, \pi_{m-1} \geq 2$ with $\pi_{m}=1$ and $m \geq 2$; or $\pi_{1}, \ldots, \pi_{j-1} \geq 2$ with $\pi_{j}=1$ and $m>j>1$. Thus, we have the following contributions $1, q_{1} x y$, 


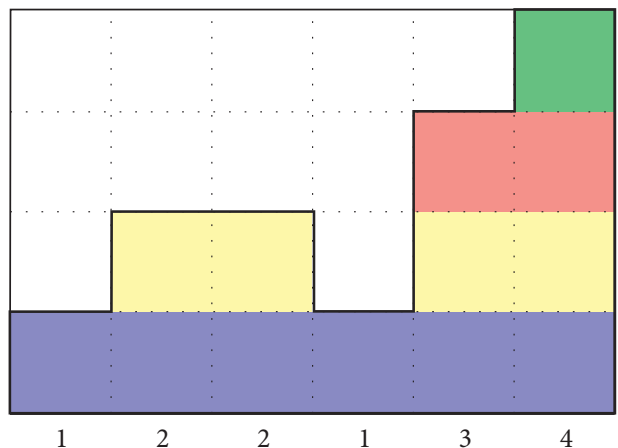

Figure 1: Composition 122134 of 13 with 5 impulses.

$x y\left(H\left(x, y ; q_{1}, q_{2}, \ldots\right)-1\right), \quad q_{1}\left(H\left(x, x y ; q_{1}, q_{2}, \ldots\right)-1\right), \quad$ respectively. Hence, $q_{1} x y\left(H\left(x, x y ; q_{1}, q_{2}, \ldots\right)-1\right)$, and

$$
x y\left(H\left(x, x y ; q_{1}, q_{2}, \ldots\right)-1\right)\left(H\left(x, y ; q_{1}, q_{2}, \ldots\right)-1\right)
$$

$$
\begin{aligned}
H\left(x, y ; q_{1}, q_{2}, \ldots\right)= & 1+q_{1} x y+x y\left(H\left(x, y ; q_{1}, q_{2}, \ldots\right)-1\right)+q_{1}\left(H\left(x, x y ; q_{2}, q_{3}, \ldots\right)-1\right) \\
& +q_{1} x y\left(H\left(x, x y ; q_{2}, q_{3}, \ldots\right)-1\right) \\
& +x y\left(H\left(x, x y ; q_{2}, q_{3}, \ldots\right)-1\right)\left(H\left(x, y ; q_{1}, q_{2}, \ldots\right)-1\right),
\end{aligned}
$$

which, by iterating, implies the following result.

Theorem 3. The generating function $H\left(x, y ; q_{1}, q_{2}, \ldots\right)$ satisfies

$$
H\left(x, y ; q_{1}, q_{2}, \ldots\right)=1-q_{1}+\frac{q_{1} H\left(x, x y ; q_{2}, q_{3}, \ldots\right)}{1-x y H\left(x, x y ; q_{2}, q_{3}, \ldots\right)} .
$$

Moreover, it is given by

$$
1-q_{1}-\frac{q_{1}}{x y-\frac{1}{1-q_{2}-\frac{q_{2}}{x^{2} y-\frac{1}{1-q_{3}-\frac{q_{3}}{x^{3} y-\frac{1}{\ddots}}}}}}
$$

Note that $H(x, y ; 1,1, \ldots)=1 /(1-(x y /(1-x)))$ satisfies $\quad H(x, y ; 1,1, \ldots)=H(x, x y ; 1,1, \ldots) /(1-x y H$ $(x, x y ; 1,1, \ldots))$. Define $H_{m}(x, y ; q)=H\left(x, y ; q_{1}, q_{2}, \ldots\right)$ with $q_{m}=q$ and $q_{j}=1$ for all $j \neq m$. Then, Theorem 3 gives the following result.

Theorem 4. The generating function $H_{m}(x, y ; q)$ is given by

$$
H_{m}(x, y ; q)=\frac{H_{m-1}(x, x y ; q)}{1-x y H_{m-1}(x, x y ; q)},
$$

where

$$
\begin{aligned}
H_{1}(x, y ; q)= & 1-q+\frac{q H(x, x y ; 1,1, \ldots)}{1-x y H(x, x y ; 1,1, \ldots)}=1-q \\
& +\frac{q}{1-(x y /(1-x))} .
\end{aligned}
$$

Define $\left.(\mathrm{d} / \mathrm{d} q) H_{m}(x, y ; q)\right|_{q=1}$ to be the generating function $H_{m}^{\prime}(x, y)$. Then, Theorem 4 (and induction on $m$ ) shows that for all $m \geq 1$,

$$
H_{m}^{\prime}(x, y)=\frac{\left(x^{m} y /(1-x)\right)}{\left(1-\left(x^{m} y /(1-x)\right)\right)(1-(x y /(1-x)))^{2}} .
$$

Hence, $H_{m}^{\prime}(x, 1)=\left(x^{m}\left(1-x-x^{m}\right)\right) /(1-2 x)^{2}$, which leads to the following result.

Corollary 3. Let $n \geq m \geq 1$. Then, the total number of impulses at level $m$ over all bargraphs of $n$ is given by

$$
(n-m+1) 2^{n-m}-(n-m) 2^{n-m-1}-(n-2 m+1) 2^{n-2 m} \text {. }
$$

When $m=1$, this gives $2^{n-1}$ as expected since $I_{1}(\pi)=1$ for every compositions of $n$.

As another application, we consider the generating function $J_{m}(x, y)=H\left(x, y ; q_{1}, q_{2}, \ldots\right)$ with $q_{j}=0$ for $j \geq m$ and $q_{j}=1$ for all $j<m$. Note that $J_{m}(x, y)$ is the generating 
function for the number of compositions of $n$ with exactly $m$ parts such that each part is at most $m-1$. Theorem 3 shows $A_{m}(x, y)=\left(A_{m-1}(x, x y) /\left(1-x y A_{m-1}(x, x y)\right)\right) \quad$ with $A_{1}(x, y)=1$. Hence, $\quad A_{m}(x, y)=1 /(1-$ $\left(x+x^{2}+\cdots+x^{m-1}\right) y$ ), for all $m \geq 1$, as it is well known (see $[6])$.

\section{Data Availability}

No data were used to support this study.

\section{Conflicts of Interest}

The authors declare that they have no conflicts of interest.

\section{Acknowledgments}

This study was supported by the National Research Foundation under grant numbers 89147, 86329, and 81021.

\section{References}

[1] M. Archibald, A. Blecher, C. Brennan, A. Knopfmacher, and T. Mansour, "Shedding light on words," Applicable Analysis and Discrete Mathematics, vol. 11, no. 1, pp. 216-231, 2017.

[2] A. Blecher and T. Mansour, "Counting staircases in integer compositions," Online Journal of Analytic Combinatorics, vol. 11, no. 11, 2016.

[3] T. Mansour and A. S. Shabani, "Interior vertices and edges in bargraphs," Notes on Number Theory and Discrete Mathematics, vol. 25, no. 2, pp. 181-189, 2019.

[4] T. Mansour and M. Shattuck, "Combinatorial parameters on bargraphs of permutations," Transactions on Combinatorics, vol. 7, no. 2, pp. 1-16, 2018.

[5] M. Bousquet-Mélou and A. Rechnitzer, "The site-perimeter of bargraphs," Advances in Applied Mathematics, vol. 31, no. 1, pp. 86-112, 2003.

[6] S. Heubach and T. Mansour, Combinatorics of Compositions and Words, Discrete Mathematics and its Applications, CRC Press, Taylor and Francis Group, Boca Raton, FL, USA, 2010.

[7] P. Flajolet and R. Sedgewick, Analytic Combinatorics, Cambridge University Press, Cambridge, UK, 2009. 